\title{
Resistência antimicrobiana associada em isolados clínicos de Enterococcus spp.
}

\author{
Associated antimicrobial resistance in Enterococcus spp. clinical isolates
}

\author{
Cláudia Castelo Branco Artiaga Kobayashi ${ }^{1,2}$, Geraldo Sadoyama ${ }^{3}$, José Daniel Gonçalves Vieira ${ }^{3}$ e \\ Fabiana Cristina Pimenta ${ }^{2,3}$
}

\section{RESUMO}

Introdução: $\mathrm{O}$ aumento da prevalência de isolados de enterococos em hospitais, particularmente Enterococcus resistente à vancomicina (VRE), é importante por causa da limitada terapia antimicrobiana efetiva para o tratamento de infecções enterocócicas. Métodos: O presente trabalho apresentou uma investigação retrospectiva de dados de suscetibilidade in vitro quantitativa para uma variedade de antimicrobianos frente aos isolados de Enterococcus spp. e avaliação da associação de resistência entre os agentes antimicrobianos apontados como escolha para o tratamento de infecções causadas por VRE, através do cálculo do risco relativo. Resultados: Dos 156 isolados de enterococos, 40 (25,6\%) foram resistentes a três ou mais antimicrobianos, incluindo $7,7 \%(\mathrm{n}=12 / 156)$ resistentes à vancomicina. A associação de resistência elevada foi mais pronunciada entre os isolados de VREs com antimicrobianos alternativos e primários para o tratamento de infecções causadas por estes patógenos, incluindo ampicilina $(100 \%, R R=7,2)$, estreptomicina $(90,9 \%, R R=4,9)$, rifampicina $(91,7 \%$, $\mathrm{RR}=3,1)$ e linezolida $(50 \%, \mathrm{RR}=11,5)$, apesar da alta taxa de suscetibilidade a esta droga $(94,9 \%)$. Conclusões: A resistência associada significativa aos antimicrobianos de primeira escolha e alternativos, usados no tratamento de infecções graves por cepas com o fenótipo VRE e que requerem um regime terapêutico combinado, evidencia alternativas terapêuticas ainda mais limitadas na instituição analisada.

Palavras-chaves: Enterococcus spp. Resistência à vancomicina. Resistência antimicrobiana associada.

\begin{abstract}
Introduction: The increasing prevalence of enterococci strains in hospitals, particularly among isolates of vancomycin-resistant enterococci (VRE), poses important problems because of the limited effect of antimicrobial therapy for enterococcal infections. Methods: This work presents a retrospective investigation of quantitative in vitro susceptibility data for the range of antimicrobials against Enterococcus spp. isolates and evaluation of the association of resistance between antimicrobial agents recommended as the treatment of choice for infections caused by VRE through calculation of the relative risk. Results: Of the 156 enterococci isolates, 40 (25.6\%) were resistant to 3 or more antimicrobials, including $7.7 \%(n=12 / 156)$ vancomycin resistant. The association of elevated resistance was more pronounced among VRE isolates against alternative and primary antimicrobials for the treatment of infections caused by these pathogens, including ampicillin $(100 \%, R R=7.2)$, streptomycin $(90.9 \%, R R=4.9)$, rifampin $(91.7 \%, \mathrm{RR}=3.1)$ and linezolid $(50 \%, \mathrm{RR}=11.5)$, despite high susceptibility to this drug (94.9\%). Conclusions: The significant associated resistance to alternative and first choice antimicrobials used in the treatment of serious infections of strains with the VRE phenotype and that require a combined therapeutic regime, revealed even more limited therapeutic alternatives in the institution analyzed.
\end{abstract}

Keywords: Enterococcus spp. Vancomycin resistance. Associated antimicrobial resistance.

1. Laboratório de Microbiologia, Hospital de Urgências de Goiânia, Goiânia, GO. 2. Programa de PósGraduação em Ciências da Saúde, Universidade Federal de Goiás, Goiânia, GO. 3. Instituto de Patologia Tropical e Saúde Pública, Universidade Federal de Goiás, Goiânia, GO.

Endereço para correspondência: Dr. Geraldo Sadoyama. IPTSP/UFG. Rua $235 \mathrm{~s} / \mathrm{n}$, Laboratório de Bacteriologia Médica, Sala 418, Setor Universitário, 74605-050 Goiânia, GO.

Tel: 5562 3209-6108

e-mail: gsadoyama@yahoo.com.br

Recebido para publicação em 22/06/2010

Aceito em 11/01/2011

\section{INTRODUÇÃO}

Enterococcus spp. tem apresentado uma importância crescente, na etiologia das infecções hospitalares, por ser considerado um microrganismo intrinsecamente resistente às drogas comumente utilizadas, resultando em um grande desafio clínico ${ }^{1}$. Além disso, a emergência de Enterococcus spp. resistente à vancomicina (VRE), opção de reserva para tratamento de infecções sérias por enterococos multirresistentes, tem sido alarmante para os infectologistas. Isso se deve não só à restrita opção para o tratamento das infecções, mas à dificuldade de se limitar a disseminação nosocomial, principalmente por contato direto, quando do estabelecimento deste microrganismo no ambiente hospitalar ${ }^{2}$. Dessa forma, a emergência e a propagação Enterococcus spp. resistente à vancomicina é uma grande ameaça associada a um aumento da morbidade e mortalidade. A alta prevalência de casos envolve a resistência não só à vancomicina, mas aos novos antimicrobianos, o que torna a terapêutica ainda mais limitada ${ }^{3}$.

Um dos problemas da resistência individual dos enterococos às múltiplas drogas consiste na associação elevada de resistência a outros antimicrobianos, particularmente entre isolados VRE, nos quais o risco de resistência associada entre as opções terapêuticas alternativas de infecções graves, incluindo ampicilina, altas concentrações de aminoglicosídeos e linezolida tem sido comumente relatado ${ }^{4,5}$. Em decorrência da seleção de enterococos multirresistentes em estabelecimentos clínicos, e da presença de resistência associada, compromete ainda mais o controle das infecções causadas por estes patógenos, torna-se necessário conhecer o grau dessa resistência entre os diferentes agentes antimicrobianos, principalmente aqueles que têm sido apontados como opções terapêuticas primária ou alternativa para os casos mais graves de infecções causadas por VRE. 


\section{MÉTODOS}

Foi realizada uma análise retrospectiva de dados relacionados aos testes de suscetibilidade antimicrobiana dos isolados de Enterococcus spp., no período compreendido entre janeiro de 2006 a dezembro de 2008. Os microrganismos foram provenientes dos espécimes clínicos de pacientes internados em um hospital público de Goiânia, referência em urgência e emergência.

A identificação inicial destes patógenos foi realizada através de métodos convencionais (manuais), de acordo com o Manual de Microbiologia Clínica para o Controle de Infecção em Serviços de Saúde ${ }^{6}$ e dos VREs confirmada através do método automatizado que utiliza painéis de microdiluição contendo substratos para a identificação do isolado bacteriano (Micro scan4 - Dade Behring ${ }^{\circledR}$ ). Todos os testes de suscetibilidade foram realizados através do método de disco-difusão, segundo normas padronizadas pelo documento M2-A8, suplementado pelo M100-S19 do National Committee for Clinical Laboratory Standards (NCCLS)/Clinical and Laboratory Standard Institute (CLSI) ${ }^{7,8}$. Todos os isolados foram categorizados para suscetibilidade aos seguintes agentes antimicrobianos: norfloxacina $(10 \mu \mathrm{g})$, ciprofloxacina $(5 \mu \mathrm{g})$, penicilina $(10 \mathrm{U})$, ampicilina $(10 \mu \mathrm{g})$, vancomicina $(30 \mu \mathrm{g})$, rifampicina $(5 \mu \mathrm{g})$, gentamicina $(120 \mu \mathrm{g})$, estreptomicina $(300 \mu \mathrm{g})$, tetraciclina $(30 \mu \mathrm{g})$ e linezolida $(30 \mu \mathrm{g})$. Enterococcus faecalis ATCC 29212 foi incluída como cepa controle de qualidade ${ }^{7}$.

Para cada espécie, a resistência antimicrobiana a uma droga foi calculada na presença e ausência de resistência a outra droga analisada. A taxa de resistência foi calculada como o número de isolados intermediários e resistentes divididos pelo total de isolados. A resistência cruzada foi definida como a resistência aumentada a dois ou mais antimicrobianos dentro da mesma classe e a resistência associada foi determinada pela resistência aumentada a duas ou mais drogas de diferentes classes ${ }^{9}$.
A resistência associada foi quantificada calculando-se o RR (relative risk) com diferenças significantes quando $p<0,05$, através do teste $\mathrm{x}^{2}$ ou exato de Fisher, utilizando-se o software Epi Info v. 2000, CDC.

\section{Considerações éticas}

O presente trabalho foi aprovado pelo Comitê de Ética em Pesquisa do Hospital de Urgências de Goiânia, atendendo a resolução 196/96, sob o protocolo CEP/HUGO/SES N084/08.

\section{RESULTADOS}

Do total de 5.440 bactérias isoladas no período compreendido entre janeiro de 2006 e dezembro de 2008, foram identificadas 156 cepas de Enterococcus spp., as quais foram provenientes dos espécimes clínicos: urina (95), secreções de feridas cirúrgicas, intraabdominais, escaras, abscessos, drenos (42), sangue (9), líquidos corporais (3), tecido e fragmentos ósseos (5) e ponta de cateter (2). Entre estes isolados, $40(25,6 \%)$ apresentaram resistência a três ou mais antimicrobianos, sendo considerados resistentes a múltiplas drogas, incluindo $12(7,7 \%)$ resistentes à vancomicina. A resistência individual verificada entre Enterococcus spp. foi mais elevada à ciprofloxacina $(47,4 \%)$, norfloxacina $(37,2 \%)$ e rifampicina $(28,8 \%)$ que à tetraciclina (16\%), ampicilina (12,8\%) e aos aminoglicosídeos de alta concentração (9,6 - 11,5\%), além da vancomicina. Por outro lado, a taxa de suscetibilidade à linezolida foi de 94,9\% (Figura 1).

Além da resistência individual, a resistência a uma determinada droga associada a um aumento da resistência a outros agentes antimicrobianos também foi verificada. A taxa e risco relativo de resistência com os perfis de resistência cruzada e associada dos isolados de Enterococcus spp. tanto resistentes como sensíveis a outros agentes antimicrobianos são mostrados nas Tabelas 1 e 2.

Ao se analisar a resistência associada entre os antimicrobianos, foi estatisticamente significante o risco de resistência aumentado

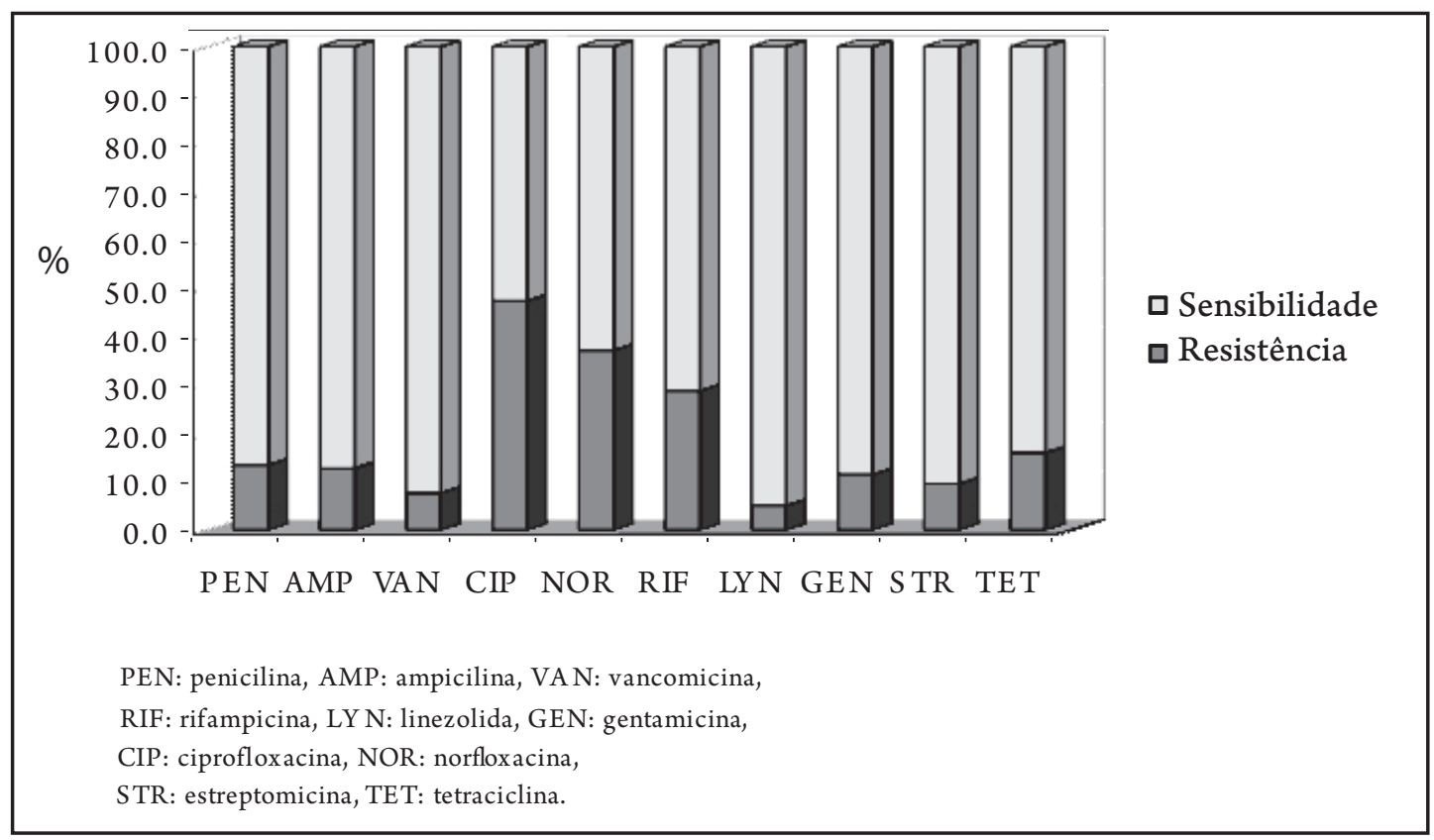

Figura 1 - Perfil de suscetibilidade antimicrobiana dos isolados de Enterococcus spp. 
TABELA 1 - Resistência cruzada e associada em Enterococcus spp. para os antimicrobianos utilizados no tratamento combinado.

\begin{tabular}{|c|c|c|c|c|c|c|c|c|c|c|c|}
\hline \multirow{3}{*}{\multicolumn{2}{|c|}{$\begin{array}{l}\text { Antimicrobianos } \\
\mathrm{n}^{\mathrm{o}} \text { de isolados }\end{array}$}} & \multicolumn{10}{|c|}{ Percentual de resistência e risco relativo } \\
\hline & & \multicolumn{2}{|c|}{ PEN } & \multicolumn{2}{|c|}{ AMP } & \multicolumn{2}{|c|}{ VAN } & \multicolumn{2}{|c|}{ GEN } & \multicolumn{2}{|c|}{ STR } \\
\hline & & \multirow{2}{*}{$\frac{\%}{100,0}$} & \multirow{2}{*}{$\frac{\mathrm{RR}}{\mathrm{NA}}$} & \multirow{2}{*}{$\begin{array}{r}\% \\
95,2\end{array}$} & \multirow{2}{*}{$\frac{\mathrm{RR}}{\mathrm{NA}}$} & \multirow{2}{*}{$\begin{array}{r}\% \\
57,1\end{array}$} & \multirow{2}{*}{$\frac{\mathrm{RR}}{\mathrm{NA}}$} & \multirow{2}{*}{$\frac{\%}{38,5}$} & \multirow{2}{*}{$\frac{\mathrm{RR}}{0,6}$} & \multirow{2}{*}{$\begin{array}{r}\% \\
81,2\end{array}$} & \multirow{2}{*}{$\frac{\mathrm{RR}}{8,9}$} \\
\hline PEN & $\mathrm{R}=21$ & & & & & & & & & & \\
\hline & $S=135$ & 0,0 & & 0,0 & & 0,0 & & 68,4 & & 9,1 & \\
\hline \multirow[t]{2}{*}{ AMP } & $\mathrm{R}=20$ & 100,0 & 50,0 & 100,0 & NA & 60,0 & NA & 38,5 & 0,6 & 81,2 & 8,9 \\
\hline & $S=50$ & 2,0 & & 0,0 & & 0,0 & & 68,4 & & 9,1 & \\
\hline \multirow[t]{2}{*}{ VAN } & $\mathrm{R}=12$ & 100,0 & 16,0 & 100,0 & 7,2 & 100,0 & NA & 30,0 & 0,4 & 90,9 & 4,9 \\
\hline & $S=144$ & 6,2 & & 13,8 & & 0,0 & & 68,2 & & 18,5 & \\
\hline \multirow[t]{2}{*}{ CIP } & $\mathrm{R}=74$ & 25,7 & NA & 46,3 & NA & 16,2 & NA & 56,5 & 1,0 & 48,1 & 2,6 \\
\hline & $S=32$ & 0,0 & & 0,0 & & 0,0 & & 55,6 & & 18,2 & \\
\hline \multirow[t]{2}{*}{ NOR } & $\mathrm{R}=58$ & 27,6 & NA & 55,2 & NA & 18,9 & NA & 63,1 & 0,9 & 52,4 & 1,6 \\
\hline & $S=31$ & 0,0 & & 0,0 & & 0,0 & & 66,7 & & 33,3 & \\
\hline \multirow[t]{2}{*}{ RIF } & $\mathrm{R}=45$ & 31,1 & 3,7 & 63,6 & 4,8 & 24,4 & 20,5 & 35,3 & 0,4 & 68,4 & 6,5 \\
\hline & $S=84$ & 8,3 & & 13,3 & & 1,2 & & 80,0 & & 10,5 & \\
\hline \multirow[t]{2}{*}{ LYN } & $\mathrm{R}=8$ & 75,0 & 2,9 & 75,0 & 2,9 & 75,0 & 6,2 & 50,0 & 0,9 & 75,0 & 2,4 \\
\hline & $S=50$ & 26,0 & & 26,0 & & 12,0 & & 58,3 & & 31,0 & \\
\hline \multirow[t]{2}{*}{ GEN } & $\mathrm{R}=18$ & 27,8 & 0,5 & 27,8 & 0,5 & 16,7 & 0,3 & 100,0 & NA & 22,2 & 0,3 \\
\hline & $S=14$ & 57,1 & & 57,1 & & 50,0 & & 0,0 & & 64,3 & \\
\hline \multirow[t]{2}{*}{ STR } & $\mathrm{R}=15$ & 86,7 & 6,6 & 86,7 & 6,6 & 66,7 & 15,3 & 30,7 & 0,4 & 100,0 & NA \\
\hline & $S=23$ & 13,0 & & 13,0 & & 4,3 & & 73,7 & & 0,0 & \\
\hline \multirow[t]{2}{*}{ TET } & $\mathrm{R}=25$ & 28,0 & 0,5 & 29,2 & 0,6 & 12,0 & 0,3 & 70,6 & 1,7 & 16,7 & 0,3 \\
\hline & $S=21$ & 52,4 & & 52,4 & & 42,9 & & 40,0 & & 61,1 & \\
\hline
\end{tabular}

PEN: penicilina, AMP: ampicilina, VAN: vancomicina, CIP: ciprofloxacina, NOR: norfloxacina, RIF: rifampicina, LYN: linezolida, GEN: gentamicina alto nível, STR: estreptomicina alto nível, TET: tetraciclina, RR: risco relativo,

NA: não aplicável, R: número de isolados resistentes, $\mathrm{S}$ : número de isolados sensíveis.

TABELA 2 - Resistência cruzada e associada em Enterococcus spp. para quinolonas, rifamicina, oxazolidinona e tetraciclina.

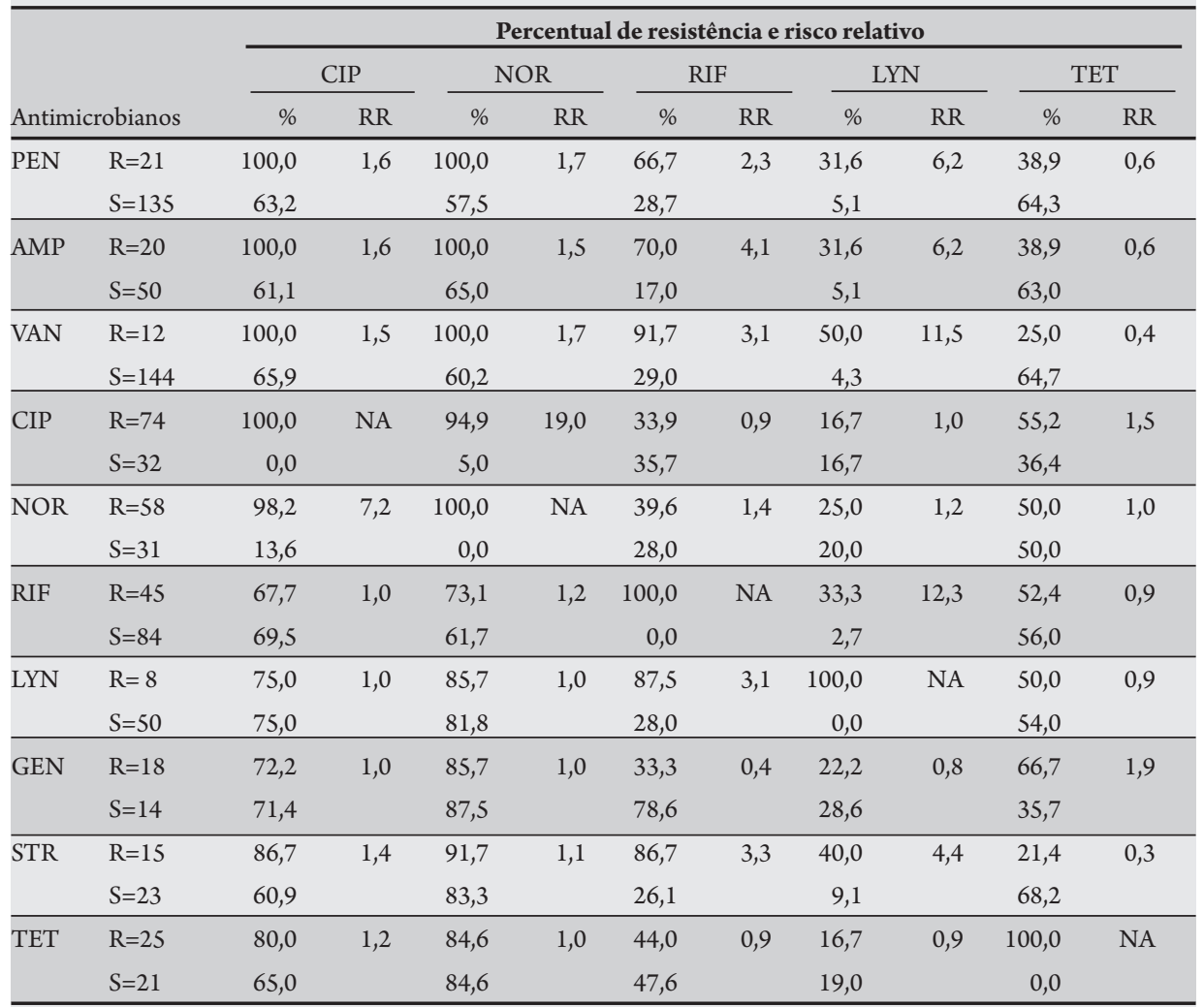

PEN: penicilina, AMP: ampicilina, VAN: vancomicina, CIP: ciprofloxacina, NOR: norfloxacina, RIF: rifampicina, LYN: linezolida, GEN: gentamicina alto nível, STR: estreptomicina alto nível, TET: tetraciclina, RR: risco relativo,

NA: não aplicável, R: número de isolados resistentes, S: número de isolados sensíveis. entre os isolados resistentes, exceto à gentamicina de alta concentração e às tetraciclinas, a qual foi mais evidente em isolados sensíveis. Assim, a resistência à penicilina ocorreu em $100 \%$ nos isolados resistentes à ampicilina e à vancomicina, com um elevado risco relativo ( 16 a 50), mas somente em $2 \%$ e $6,2 \%$ nas cepas sensíveis, respectivamente. A resistência à rifampicina foi de 91,7\% e 87,5\% em isolados resistentes à vancomicina e à linezolida, comparado com $29 \%$ e $28 \%$ em sensíveis, respectivamente. Associação elevada foi verificada com as opções terapêuticas ampicilina, estreptomicina, rifampicina e linezolida de infecções graves causadas por enterococos resistentes. A resistência à penicilina, ampicilina, vancomicina, linezolida e às altas concentrações de aminoglicosídeos apresentou risco relativo estatisticamente significante entre os isolados resistentes à rifampicina que nos sensíveis $(\mathrm{p}<0,05)$. Similarmente, foi verificada uma resistência pronunciada à estreptomicina de alta concentração em isolados resistentes às penicilinas, vancomicina, rifampicina e à linezolida (superior a 48\%), com uma maior taxa em isolados resistentes à vancomicina $(90,9 \%)$. Observou-se também uma resistência associada entre ciprofloxacina, estreptomicina, rifampicina, vancomicina e linezolida em isolados resistentes à ampicilina (100\%, 81,2\%, 70\%, 60\%, $31,6 \%$ resistentes), sendo estatisticamente significante $(\mathrm{p}<0,05)$ a diferença na suscetibilidade a estas drogas entre os isolados resistentes e sensíveis.

Com relação aos isolados de VRE, notou-se uma associação com a resistência aumentada à ampicilina $(100 \%, R R=7,2)$, estreptomicina (90,9\%, RR $=4,9)$, rifampicina $(91,7 \%$, $\mathrm{RR}=3,1)$ è̀ linezolida $(50 \%, \mathrm{RR}=11,5)$, apesar da maior sensibilidade a esta droga.

\section{DISCUSSÃO}

A frequência de VRE foi semelhante a alguns estudos de vigilância microbiológica em países europeus e norte-americanos, os quais demonstraram taxas entre $2,3 \%$ e $7,6 \%^{10}$. Entretanto, Castanheira cols ${ }^{5}$, ao analisarem a tendência de VRE nos últimos dez anos na América do Norte, observaram taxas de 3,3\% e $70 \%$ de Enterococcus faecalis e Enterococcus faecium resistentes à vancomicina, respectivamente nos EUA, 
e menores que $1 \%$ no Canadá. Na América Latina e no Brasil, tem sido observado um importante aumento, com o percentual situando-se em torno de $2 \%$ e $7 \%$, respectivamente ${ }^{11,12}$. No entanto, em São Paulo, estudos têm demonstrado um aumento progressivo na incidência de Enterococcus spp. resistente à vancomicina (9,5\% em 2000 a 15,8\% em 2002), evidenciando um problema crescente no país e uma situação preocupante pelo rápido perfil de endemicidade desse microrganismo nesse estado ${ }^{2}$. Segundo alguns autores, essa emergência de enterococos multirresistentes é um grande desafio à terapia ${ }^{13}$, e tem sido paralela à ocorrência de $\mathrm{VRE}^{14}$, cujos fatores de risco para colonização ou infecção tem sido o uso parenteral de vancomicina e de cefalosporinas de $3^{\mathrm{a}}$ geração ${ }^{15}$.

A alta taxa de suscetibilidade à linezolida indica que os níveis de atividade desta droga permanecem altos na instituição estudada. Segundo a literatura, a resistência à linezolida tem sido relatada com ocorrência esporádica e incomum entre os enterococos ${ }^{16}$. Apesar de raro, o surgimento dessa resistência tem sido associado com o aumento da utilização da linezolida durante a terapia ${ }^{17,18}$. Segundo Dobbs cols ${ }^{17}$, a exposição a outros antimicrobianos, particularmente fluoroquinolonas, pode ser importante também para a emergência de resistência à linezolida. Estes pesquisadores observaram resistência aumentada à ciprofloxacina quase $100 \%$ em isolados de VRE resistentes à linezolida. No presente trabalho, $50 \%$ dos isolados de VRE resistentes à linezolida apresentaram resistência aumentada à ciprofloxacina. A presença dessa resistência associada compromete ainda mais o controle clínico das infecções causadas por estes patógenos. Uma vez que a linezolida tem sido considerado um dos novos antimicrobianos úteis para o tratamento de infecções mais graves, incluindo urinárias, de pele e tecido mole e bacteremia devido $\mathrm{VRE}^{19}$, a observação do uso de ciprofloxacina como pré-requisito para o desenvolvimento de resistência à linezolida limita consideravelmente as opção terapêuticas disponíveis ${ }^{17}$. Além do mais, a demonstração no presente estudo da resistência à linezolida em torno de 6 a 12 vezes mais comum em isolados resistentes à ampicilina e vancomicina, mostra que esse antimicrobiano deve ser utilizado com cautela, principalmente no tratamento das infecções em que há altas taxas de resistência à ampicilina, como ocorre com Enterococcus faecium ${ }^{4}$.

No presente trabalho, foi verificado que a resistência à ampicilina foi mais associada com a resistência aumentada à vancomicina do que entre as demais drogas, evidenciando maior risco de resistência associada entre ambas às drogas (risco relativo de 7,2). Estas taxas foram semelhantes aos dados obtidos por Bettcher ${ }^{4}$, em análise do perfil de sensibilidade das cepas de Enterococcus faecium e Enterococcus faecalis resistentes à vancomicina, com exceção apenas para a maior sensibilidade às penicilinas ( $87 \mathrm{a} 93 \%)$ verificada entre os isolados desta última espécie. $\mathrm{Oh} \mathrm{cols}^{20}$, em um estudo de vigilância multicêntrico na Coréia, verificaram taxas de resistência significativamente mais altas à ampicilina (100\%), tetraciclina (15,9\%) e ciprofloxacina (100\%) entre os isolados de Enterococcus resistentes à vancomicina (Enterococcusfaecium) que entre as cepas sensíveis (91,4\%, 5,9\% e 92,5\%, respectivamente) ao compararem a resistência antimicrobiana entre esses dois fenótipos. Maiores taxas de resistência à ampicilina (13\%), ciprofloxacina (95\%), gentamicina e estreptomicina de alta concentração (20 e 40\%, respectivamente) entre os isolados de VRE comparados aos isolados sensíveis à vancomicina foram detectadas por Castanheira cols ${ }^{5}$.

A resistência adicional a múltiplos antibióticos, incluindo ampicilina e a altas concentrações de aminoglicosídeos é conhecidamente expressa por Enterococcus spp. resistente à vancomicina ${ }^{14}$. Estudos têm demonstrado uma associação entre resistência à ampicilina e vancomicina, particularmente entre isolados de Enterococcus faecium, nos quais a resistência à ampicilina é frequentemente precedente à vancomicina ${ }^{13}$. Esta associação se deve à ligação genética entre excessiva produção de proteínas de ligação à penicilina de baixa afinidade (PBP 5) ${ }^{21}$ e ambos antibióticos e ao uso prévio de $\beta$-lactâmicos, tais como cefalosporinas, como fator predisponente para a emergência de enterococos resistentes à vancomicina ${ }^{15}$.Já a resistência a altas concentrações de aminoglicosídeos é usualmente mediada por enzimas modificadoras de aminoglicosídeos, sendo disseminada com alta frequência entre enterococos ${ }^{22}$. O elevado nível de resistência dos enterococos aos aminoglicosídeos neutraliza o efeito bactericida resultante da associação com $\beta$-lactâmicos e glicopeptídeos, diminuindo, portanto, a eficácia desta associação contra estes patógenos. Dessa forma, Enterococcus resistente à vancomicina, particularmente Enterococcusfaecium, representa um grande problema terapêutico, especialmente quando combinado com resistência a glicopeptídeo e aminoglicosídeo ${ }^{23}$. Em função da ausência do efeito bactericida, as altas taxas de resistência à ampicilina e às altas concentrações de estreptomicina demonstradas neste estudo podem dificultar o tratamento das infecções mais graves causadas por cepas de enterococos resistentes na instituição analisada. Além disso, a associação de resistência elevada verificada com linezolida, rifampicina e estreptomicina de alta concentração, consideradas opções terapêuticas usadas no tratamento de infecções graves por cepas com o fenótipo VRE e que requerem um regime terapêutico combinado ${ }^{22}$, evidencia alternativas terapêuticas ainda mais limitadas na instituição analisada.

O uso da terapia antimicrobiana múltipla ou prolongada de vancomicina e de outros agentes antimicrobianos que podem influir no desenvolvimento de VRE, incluindo $\beta$-lactâmicos, fluoroquinolonas ${ }^{1,24}$ e outras drogas para as quais VRE é resistente podem potencialmente fornecer uma vantagem seletiva para este agente, o que aumenta a sua sobrevivência no ambiente hospitalar ${ }^{25}$, dificultando assim a prevenção da disseminação deste microrganismo.

A associação da resistência elevada verificada entre antimicrobianos alternativos e de primeira escolha para o tratamento de VRE ratifica a necessidade de maior cautela com relação à indicação correta de um antimicrobiano, principalmente daquele cuja administração poderá causarumapressão seletiva para enterococosmultirresistentes, ampliando a possibilidade de falha na terapia empírica primária e alternativa.

\section{CONFLITO DE INTERESSE}

Os autores declaram não haver nenhum tipo de conflito de interesse no desenvolvimento do estudo.

\section{REFERÊNCIAS}

1. Martinez JA, Ruthazer R, Hansjosten K, Barefoot L, Snydman DR. Role of environmental contamination as a risk factor for acquisition of vancomycinresistant enterococci in patients treated in a medical intensive care unit. Arch Intern Med 2003; 163:1905-1912.

2. Furtado GHC, Martins ST, Coutinho AP, Soares GMM, Wey SB, Servolo EA Incidência de Enterococcus resistente à vancomicina em hospital universitário no Brasil. Rev Saude Publica 2005; 39:41-46.

3. Sung JML, Lindsay JA. Staphylococcus aureus Strains That are Hypersusceptible to Resistance Gene Transfer from Enterococci. Antimicrob Agents Chemother 2007; 51:2189-2191.

4. Bettcher L. O paciente portador de Enterococcus resistente à vancomicina em um hospital público de Belo Horizonte: aspectos demográficos, epidemiológicos 
e microbiológicos [dissertação]. [Belo Horizonte]: Escola de Enfermagem. Universidade Federal de Minas Gerais; 2008. 104p.

5. Castanheira M, Biednlach D, Sader H, Fritsche T, Jones R. Prevalence of vancomycin-resistant Enterococcus and associated resistance patters among isolates of SENTRY Program surveillance in North America. Poster session presented at: $18^{\text {th }}$ European Congress of Clinical Microbiology and Infectious Diseases; 2008; Barcelona, Spain.

6. Levy CE, Von Nowakonski A, Mendes CMF, Oplustil C, Zoccoli CM, Marffei CM, et al. Manual de Microbiologia Clínica para o Controle de Infecção em Serviços de Saúde. In: Módulo V. Detecção e identificação das bactérias de importância médica. Brasília: Agência Nacional de Vigilância Sanitária. Ministério da Saúde; 2004.

7. Clinical and Laboratory Standard Institute. Performance standards for antimicrobial susceptibility testing. Pennsylvania: Clinical and Laboratory Standard Institute; 2009. Nineteenth informational supplement: M100-S19.

8. National Committee for Clinical Laboratory Standards. Performance Standards for Antimicrobial Disk Susceptibility Tests. Pennsylvania: National Committee for Clinical Laboratory Standards; 2003. Approved Standard. Eighth Edition: M2-A8.

9. Kobayashi CCBA, Sadoyama G, Vieira JDG. Determinação da resistência antimicrobiana associada em isolados clínicos de Staphylococcus aureus e Pseudomonas aeruginosa em um hospital público de Goiânia, Estado de Goiás. Rev Soc Bras Med Trop 2009; 42:404-410.

10. Jones ME, Draghi DC, Thornsberry C, Karlowsky JA, Sahm DF, Wenzel RP. Emerging resistance among bacterial pathogens in the intensive care unit-a European and North America surveillance study (2000-2002). Ann Clin Microbiol Antimicrob 2004; 3:1-14.

11. Gales A, Sader H, Santiago K, Pignatari A, Jones R. Frequency and antimicrobial susceptibility profile of vancomycin-resistant enterococci from Latin America: a report from the SENTRY Antimicrobial Surveillance Program (1997- 2006). Poster session presented at: $18^{\text {th }}$ European Congress of Clinical Microbiology and Infectious Diseases; 2008; Barcelona, Spain.

12. Sader HS, Jones RN, Gales AC, Silva JB, Pignatari ACC. SENTRY Participants Group (Latin America). SENTRY antimicrobial surveillance program report: Latin American and Brazilian results for 1997 through 2001. Braz J Infect Dis 2004; 8:25-79.

13. Mundy LM, Sahm DF, Gilmore M. Relationships between Enterococcal Virulence and Antimicrobial Resistance. Clin Microbiol Rev 2000; 13:513-522.

14. Schouten MA, Hoogkamp-Korstanje JAA, Meis JFG, Voss A, The European VRE Study Group. Prevalence of vancomycin-resistant enterococci in Europe. Eur J Clin Microbiol Infect Dis 2000; 19:816-822.

15. Dahms RA, Johnson EM, Statz CL, Lee JT, Dunn DL, Beilman GJ. Third generation cephalosporins and vancomycin as risk factors for postoperative vancomycin-resistant Enterococcus infection. Arch Surg 1998; 133:1343-1346.

16. Draghi DC, Sheehan DJ, Hogan P, Sahm DF. In vitro activity of linezolid against key gram-positive organisms isolated in the United States: Results of the LEADER 2004 surveillance program. Antimicrob Agents Chemother 2005; 49:5024-5032.

17. Dobbs TE, Patel M, Waites KB, Moser SA, Stamm AM, Hoesley CJ. Nosocomial spread of Enterococcus faecium resistant to vancomycin and linezolid in a tertiary care medical center. J Clin Microbiol 2006; 44:3368-3370.

18. Scheetz MH, Knechtel SA, Malczynski M, Postelnick MJ, Qi C. Increasing incidance of linezolid-intermediate ou -resistant, vancomycin-resistant Enterococcus faecium strains parallels increasing linezolid consumption. Antimicrob Agents Chemother 2008; 52:2256-2259.

19. Kauffman CA. Therapeutic and preventative options for the management of vancomycin-resistant enterococcal infections. J Antimicrob Chemother 2003; 51:iii23-iii30.

20. Oh WS, Ko KS, Song JH, Lee MY, Park S, Peck KR, et al. High rate of resistance to quinupristin-dalfopristin in Enterococcus faecium clinical isolates from Korea. Antimicrob Agentes Chemother 2005; 49:5176-5178.

21. Ligozzi M, Pittaluga F, Fontana R. Modification of penicillin-binding protein 5 associated with high-level ampicillin resistance in Enterococcus faecium. Antimicrob Agents Chemother 1996; 40:354-357.

22. Centikaya Y, Falk P, Mayhall CG. Vancomycin-resistant enterococci. Clin Microbiol Rev 2000; 13:686-707.
23. Mohn SC, Ulvik A, Jureen R, Willems RJL, Top J, Leavis H, et al. Duplex realtime PCR assay for rapid detection of ampicillin-resistant Enterococcus faecium. Antimicrob Agents Chemother 2004; 48:556-560.

24. Cunha B. Methicillin-resistant Staphylococcus aureus/vancomycin-resistant Enterococci colonization and infection in the critical care unit. In: Infectious Diseases. $2^{\text {nd }}$ ed. New York (USA): Critical Care Medicine; 2006. 32p.

25. Murray BE. Vancomycin-resistant enterococci. Am J Med 1997; 101:284-293. 